\title{
Integrating family planning with genitourinary medicine: Developing an holistic sexual health clinic in Eastbourne
}

\author{
Martin Jones, RGN, MSc, Clinical Nurse Specialist / Co-ordinator \\ Correspondence: Martin Jones, Sexual Health, Eastbourne and County Healthcare NHS Trust, Avenue House, The Avenue, \\ Eastbourne BN21 3XY, UK. Tel: 01323444165.
}

(Accepted May $\left.16^{\text {th }}, 2000\right)$

\begin{abstract}
Summary
The idea of providing family planning and genitourinary medicine under one roof has attracted much interest. The development of an integrated sexual health clinic in Eastbourne is described, from initial discussions between disparate parties to the emergence of a one-stop-shop, with a look to the future.
\end{abstract}

\section{Key words}

family planning, genitourinary medicine, sexual health

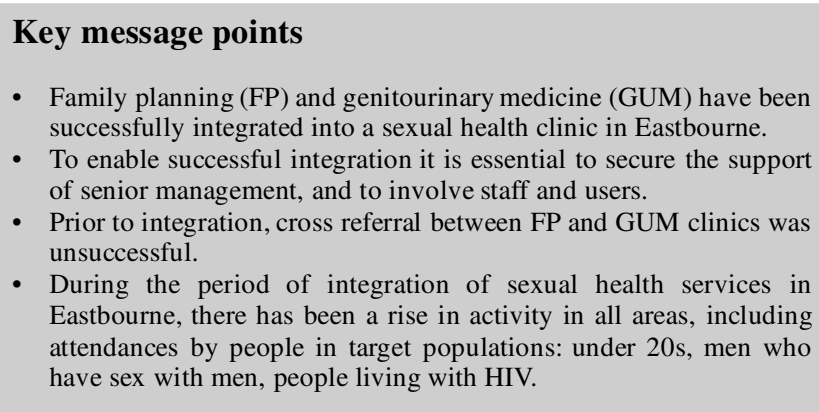

\section{Introduction}

The 1990s saw an increasing interest in, and debate about, sexual health clinics. Practical constraints in many health authorities have been described as a barrier to the integration of family planning (FP) with genitourinary medicine
(GUM), although, as a minimum, collaboration and coordination between the two services has been advocated. ${ }^{1}$ Searle identified the need for FP clinics to 'adapt or perish', listing a number of potential routes for the speciality to secure its future, including the possibility of combining with GUM. $^{2}$ The Ipswich GUM Clinic broadened its provision to include FP, drawing attention to the advantages of an holistic approach for women's sexual health.,4

Since gaining trust status, Eastbourne and County Healthcare NHS Trust had managed FP and GUM as fragmented parts of its community health services. Fortuitous historical circumstances meant that, in Eastbourne, these were held in the same building but at different times of the week. Both FP and GUM held four sessions per week, each staffed by one or two doctors with two staff nurses, plus a health adviser in the GUM sessions.

In 1994, local commissioners increased the level of funding so that a GUM consultant, shared with the neighbouring trust in Hastings, and a full-time clinical nurse specialist / co-ordinator could be appointed. A strategic review identified the disparate parts of the service, and the opportunity to bring the services more closely together was identified. This led to their integration as a sexual health service 4 years later, in July 1998. The process of achieving these changes is presented in order to encourage debate and discussion amongst colleagues within the speciality. We acknowledge that we are not the first or only sexual health clinic in the UK.

Table 1 SWOT Analysis 1995

Strengths

- FP and GUM share premises

- FP and GUM are managed by the same trust

- Some medical and nursing staff work in both FP and GUM

- Service users favour an holistic approach to sexual health

- Staff are committed to developing an holistic sexual health clinic

- Commissioners are supportive

- High level of training and role development in FP nursing

- Provision of specialist training for doctors and nurses

- Location in town centre

- Partnerships with school nursing, drugs team, youth services, etc.
Weaknesses

- Patients who present in FP and who also need GUM (and vice versa) are required to re-attend, and often do not

- Knowledge and role of nurses in GUM under-developed

- Activity above contract level

- Overspending in several budget areas

- No budget for newer contraceptive methods such as Mirena

- Reliance on volunteers, bank and sessional staff

- No telephone access for the public out of clinic times

- Lack of publicity about the clinics

- Premises unsuited to clinical work

- Doctors practising psychosexual medicine isolated from other psychosexual practitioners

Threats

- Budgetary uncertainty

- The potential removal of GUM to the hospital

- Potential failure to seize the existing opportunity through lack of vision
- Potential for integrating FP with GUM

- Trust plans to redevelop premises

- Developing links with other professionals, e.g.: general practitioners obstetricians and gynaecologists pathology

health promotion specialists

health adviser in drugs team etc. 


\section{Political factors}

- Health of the Nation targets

- Numerous strategies and needs assessments carried out by the health authority

- Report of the Working Group to examine Workloads in Genitourinary Medicine Clinics (The Monks Report) ${ }^{11}$

Social factors

- National interest in provision of holistic sexual health services

- Decrease in the age of first coitus

- Increasing demand for sexual health services

- Raised public expectations, e.g. through The Patients' Charter

- Sexual health education and health promotion encourages people to use sexual health services
Economic factors

- Removal of ring fencing on HIV monies

- Existing overspending in FP and GUM services

- An holistic service may prove more expensive than the sum of its parts as opportunistic interventions will be possible

- The potential for spending on HIV drugs to increase

- The value of preventing expensive sequaelae of unprotected sexual intercourse, e.g.:

unplanned children

abortion

HIV infection

P.I.D.

sub fertility

cervical cancer

etc.

Technological factors

- Potential computerisation of FP activity and the potential for I.T. to increase efficiency

- Advances in HIV management and therapy

- Low sensitivity of ELISA test for Chlamydia and future potential of molecular tests

\section{Getting started}

Medical, nursing and administrative staff participated in a series of meetings which included analyses of strengths, weaknesses, opportunities and threats (SWOT analysis) and political, economic, social and technological factors (PEST analysis) (Tables 1 and 2). The potential for integrating FP with GUM was obvious as the services were already 'under one roof'. 3,4 This enabled us to agree upon the shared goal of creating an holistic sexual health department.

\section{Management involvement and strategic planning}

Senior management commitment was secured by a presentation about sexual health to a joint meeting of the Trust Board with the Senior Management Team. A sexual health strategy was developed and was adopted by the Trust in 1996. Local commissioners did not have a sexual health strategy at this time, but were supportive of the initiative. As well as developing a strategy for integrating services, the following target populations were identified: under $20 \mathrm{~s}$, men who have sex with men, and people living with HIV.

\section{Staff involvement and training}

Staff appraisal was introduced, enabling individuals to identify their personal development needs for working in an integrated department. Visits to the already-established sexual health clinic in Slough were arranged for medical, nursing and administrative staff, enabling them to discuss experiences of change and to see an integrated service in action. There were already a few nurses and one doctor working in both FP and GUM. Our aim was to increase this proportion and to ensure that staff were appropriately trained in both specialities.

During the period of transition, two FP doctors have attended the Diploma in Genitourinary Medicine course, and several nurses have completed ENB courses in GUM and/or FP. Clinical leadership in FP and GUM has remained unchanged and is provided by a senior clinical medical officer (SCMO) and a consultant, respectively. We have changed the rest of our medical staffing structure from sessional clinical medical officers in FP and clinical assistants in GUM by establishing two part-time staff grade posts in sexual health. The staff grade doctors work in both FP and GUM, as does the SCMO in FP. The majority of nurses are now qualified and skilled in both FP and GUM nursing, and skill mix review has introduced the post of nursing auxiliary to all combined sessions. However, there has been no increase in the budget and therefore the basic establishment remains part-time.

One difficulty for the emerging speciality of sexual health is that the training of doctors in FP and GUM is organised via different career routes, through separate Royal Colleges. It is difficult to identify the career path for a doctor aspiring to a future post as a consultant in sexual health. This contrasts with the situation for nurses who can obtain ENB-equivalent training in FP, GUM and HIV and AIDS as part of a diploma or degree in nursing studies.

A number of in-house training events were held, covering such topics as child protection, cervical cytology, nurse issuing of hormonal contraception under protocol, taking a sexual history, etc. The sexual-history-taking training was instrumental in helping us to agree on a common approach to holistic sexual health assessment. New clinic notes were designed in order to document this. A sexual health lunch club was started and now meets regularly, its programme including audit, journal club, case presentations and a variety of invited speakers.

\section{User involvement}

A waiting room survey was carried out in Eastbourne's FP, GUM and Youth Walk-in Clinics, and in Ipswich's Sexual Health Clinic. ${ }^{6}$ The survey sought patients' attitudes towards integrated sexual health services and identified that two-thirds of patients were in favour of integration. Patients attending the Sexual Health Clinic in Ipswich were much more likely to have discussed both contraception and sexually transmitted infections during their consultations. This was interpreted as an indication that staff in separate FP and GUM clinics tended to focus on their own speciality and not on an holistic sexual health approach.

\section{Initial audit of cross referrals}

Although the FP and GUM clinics shared premises, patients who were identified in FP sessions as needing a GUM assessment were required to re-attend during a GUM session, and vice versa. The standard expected for reattendance was $100 \%$. An audit of such cross clinic referrals found that more than half did not re-attend, meaning that the standard was not met and patients' identified sexual health needs were not being fully addressed. 


\section{Concerns}

Anecdotal evidence from Slough and Ipswich, where this was experienced, suggested that there might be a temporary fall in attendances of patients in target populations. Another concern was that, only 2 weeks after integration, we moved from our usual clinic building near the town centre to temporary accommodation, half-a-mile up the road. Most of our first 8 months as an integrated sexual health clinic were therefore spent waiting to move back to purpose-designed clinic rooms at our normal community health centre base. Posters and leaflets describing the temporary move were widely distributed, key referrers were informed and the clinic's direct telephone number was retained during the period.

There was a reduction in the numbers attending the young people's walk-in clinic which have recovered since moving back. This has been interpreted as being related to the temporary move away from a convenient town centre location rather than dissatisfaction with integrated sexual health services. In the waiting room survey, users of the Eastbourne Youth Walk-in were the most enthusiastic about integration. ${ }^{6}$ Activity levels amongst key target populations have risen during the period of planning and since integration (Table 3).

Table 3 Changing activity since integration

\begin{tabular}{lcc}
\hline & $1995-1996$ & $1999-2000$ \\
\hline Total FP attendances & 5388 & 5547 \\
Proportion of FP patients under 20 years & $24 \%$ & $29 \%$ \\
Total GUM attendances & 4501 & 5157 \\
Proportion of male GUM activity: & $13 \%$ & $19 \%$ \\
men having sex with men & 24 & 31 \\
\hline
\end{tabular}

Doctors and nurses who have worked in the department since before 1994 state that the nature of the FP work has changed, with a greater bias towards complex cases which cannot be easily handled in general practice: an appropriate shift for a specialist service. ${ }^{1}$ In order to cope with the increase in demand for the service, we have reviewed our systems for re-booking appointments. Women using oral contraception are now asked to contact the clinic when they need another appointment rather than automatically rebooking at the end of a clinic visit, and women with an IUD are given the time of their next review but are not booked, reducing the rate of non-attendance. The protocol for GUM follow-ups has been revised, changing the proportion of 'new' or first attendances from $34.3 \%$ of total attendances (1995-96) to $53.9 \%$ (1999-2000).

For doctors and nurses, there was an initial tension between dealing with a patient's main presenting issue and acting holistically: finding a balance between the one-stop approach and the pressure of time in busy clinics. This has been resolved through clinical supervision and experience of the integrated approach.

In reception there were new skills and processes to be assimilated as we run FP manually and GUM on a computer system. This has perpetuated the inconvenience of having to re-register FP patients if they use the GUM service. Issuing of contraceptives was removed from the reception to consulting rooms. The immediate period after the change was stressful for staff as they adjusted to new clinic opening times, the integrated approach and moving into temporary accommodation.

\section{Financial implications}

The trust made a considerable capital investment in excess of $£ 1$ million in the building in order to create the purpose designed health centre which houses the sexual health clinic. Since the 1994 investments in GUM, however, the core budgets for sexual health services have been merely 'rolled over' from 1 year to the next, while the HIV budget has risen to reflect the introduction of highly active antiretroviral therapy. The changes described, with the exception of a new psychosexual therapy service, have therefore been achieved within existing resources and not through new investment in the service. The increase in activity has caused considerable budget pressures.

\section{Clinic organisation}

The sexual health clinic now opens for seven combined FP and GUM sessions per week. These are appointment-only clinics with two lists: one for FP and one for GUM. Two doctors staff each session, one taking a lead in FP, the other in GUM. There are two trained nurses, a health adviser and a nursing auxiliary employed in each session. In addition there is a young people's walk-in and an IUD clinic each week, and two vasectomy operating lists per month. The focus of the young people's walk-in clinic is contraception, as this is the predominant reason for attendance. Extra funding has been secured for a weekly psychosexual therapy session, jointly run with Relate, after the earlier demise of a psychosexual medicine service due to retirement and a lack of suitable local supervision.

The clinic is advertised in local directories, via leaflets and posters, and on the Trust's website as a 'Sexual Health Clinic' dealing with 'contraception, genital infections, family planning, sexually transmitted infections, HIV and AIDS, hepatitis B vaccination, emergency contraception, pregnancy testing and Youth Walk-in Clinic'.

\section{Next steps}

Improving data collection

FP data are still collected manually, while GUM is computerised. It is our belief that some activity, particularly for patients with both FP and GUM needs, is not included in current activity returns, despite our best efforts to capture it. All-through computerisation, with the creation of a single database of sexual health clinic patients, is envisaged.

\section{Further audit}

Re-auditing the work on patients with identified sexual health needs in both FP and GUM is planned.

We believe we are now in an enhanced position to effectively respond to 'The New NHS' ${ }^{7}$ and the plethora of new strategic initiatives, including: the Social Exclusion Unit Report on teenage pregnancy, ${ }^{8}$ the BMA Foundation for AIDS standards for HIV care, ${ }^{9}$ the Chlamydia pilot and strategy (as yet unpublished), the health authority's recently launched sexual health strategy ${ }^{10}$ and the government's anticipated sexual health strategy.

\section{Conclusion}

The establishment of an integrated, one-stop-shop for sexual health in central Eastbourne has taken 5 years. We have moved from inadequate first floor rooms to a purpose designed, ground floor clinic, retaining our town centre address, which is well-known in the community. The process of evolution rather than revolution has meant that 
staff have been involved in deciding the direction and the pace of change throughout.

We hope that the publication of this account will provoke a discussion amongst colleagues who have taken, who are considering taking or who have decided against taking this route.

\section{Acknowledgements}

Ipswich and Slough Sexual Health Clinics for the inspiration.

Statements on funding and competing interests

Funding. None.

Competing interest. None declared.
References

Searle ES. Adapt or perish? The challenge to clinics. British Journal of Family Planning 1995; 21: $41-42$.

Stedman Y, Elstein M. Rethinking sexual health clinics. British Medical Journal 1995; 310: $342-343$

Greenhouse P. A sexual health service under one roof. In: Pillaye J (ed.) Sexual Health Promotion in Genitourinary Medicine Clinics. London: Health Education Authority, 1994. Greenhouse, P. Under one roof: setting up sexual health services for women. Journal of Clinical and Child Health $1994 ; 19: 228-233$.

(AGUM \& MSSD) National guideline for the management of Suppl 1): S4-S8.

Jones M. Clients express preference for one-stop sexual health shop. Nursing Times 1996; 92: 32-33.

Department of Health. The New NHS: modern, dependable. London: HMSO 1997.

Social Exclusion Unit Teenage Pregnancy London: HMSO, 1999.

BMA Foundation for AIDS Standards for NHS hospital HIV services London: BMA Foundation for AIDS, 1998 .

10 Mezzone J, Iverson A, Gaudoin J, et al. Sexual Health Strategy 1999-2002. Lewes: Eas Sussex, Brighton and Hove Health Authority, 1999.

1 Department of Health Report of the Working Group to Examine Workloads in Genitourinary Medicine Clinics (The Monks Report). London: DoH, 1988

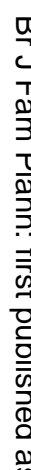

